\title{
Diagnóstico do Infarto Agudo do Miocárdio. Valor da Dosagem de Mioglobina Sérica Comparada com a Creatinofosfoquinase e sua Fração MB
}

\author{
Alexandre Biasi Cavalcanti, Roberto Henrique Heinisch, Evandro de Campos Albino, \\ João N ilson Zunino \\ Florianópolis - São José, SC
}

\begin{abstract}
Objetivo - Comparar o desempenho, em termos de teste diagnóstico, da dosagem sérica de mioglobina $(\mathrm{Mgb})$ com a creatinofosfoquinase (CK) e a sua fração $M B$ (CK-MB), para o diagnóstico de infarto agudo do miocárdio (IAM).

Métodos - Estudo observacional, contemporâneo e não controlado de 64 pacientes, admitidos entre setembro/94 e fevereiro/95, em uma emergência especializada em cardiologia, com dor torácica não traumática. Excluíram-se pacientes com sintomas há mais de 6h, trauma muscular, ressuscitação cardiopulmonar e insuficiência renal. $O$ diagnóstico de IAM foi estabelecido quando ao menos dois dos seguintes critérios estavam presentes: dor torácica típica há mais de 20min, alterações eletrocardiográficas compatíveis com necrose (ondas $Q$ ), ou elevações tardias de CK e CK-MB.
\end{abstract}

Resultados - Na amostra estudada, 18 tiveram diagnóstico de IAM. A sensibilidade (S) encontrada para $C K$, CK-MB e Mgb foi de 33\%, 22\% e $61 \%$ e a especificidade (E) de $85 \%, 96 \%$ e $98 \%$, respectivamente. A diferença entre a $\mathrm{S}$ de $\mathrm{Mgb}$ e a de CK foi de $28 \%$, com um intervalo de confiança de $95 \%$ (IC 95\%) de $-4 \%$ a 59\%, e a diferença entre a S de Mgb e a de CK-MB foi de 39\%, IC 95\% de $9 \%$ a $69 \%$. A diferença entre a E de Mgb e a de CKfoi de $13 \%$, IC $95 \%$ de $12 \%$ a $14 \%$, e a diferença entre a $E$ de $M g b$ e a de CK-MB foi de 2\%, IC $95 \%$ de -5\% a $9 \%$.

Conclusão - A mioglobina mostrou ser um marcador mais sensível e tão específico quanto a $C K-M B$, para o diagnóstico de IAM na população estudada. Em relação a CK, a Mgb foi mais específica e com igual sensibilidade.

Palavras-chave: diagnóstico do infarto agudo do miocárdio, mioglobina, creatinofosfoquinase

\section{Acute Myocardial Infarction Diagnosis. The Value of Serum Myoglobin Levels, Compared with Creatine Kinase and MB Fraction}

Purpose - To compare the diagnostic performance of two traditional plasma markers of myocardial infarction $(M I)$, creatine kinase $(C K)$ and its $M B$ fraction $(C K-M B)$, with plasma myoglobin $(\mathrm{Mgb})$ levels, for the diagnosis of MI.

Methods - From September of 94 to February of 95, in an observational, prospective, and non-controlled fashion, 64 patients admitted to a cardiology emergency room (ER), with non-traumatic chest pain were studied. Patients presenting with more than 6h after the onset of symptoms, muscular trauma, cardiopulmonary resuscitation and renal insufficiency were not included. Definitive MI diagnosis was established if the patient had at least two of the three classic MI findings: ischemic chest discomfort longer than 20min, electrocardiogram with necrosis ( $Q$ waves) and elevated $C K$ and $C K-M B$ levels.

Results - There were 18 patients with MI. The sensitivity for $C K, C K-M B$ and $M g b$ were respectively $33 \%, 22 \%$ and $61 \%$. The specificity was $85 \%$, for $C K, 95 \%$ for $C K-M B$ and $97 \%$ for $M g b$. The difference between $M g b$ and $C K$ sensitivities was $28 \%$, with a $95 \%$ confidence interval (CI) from $-4 \%$ to $59 \%$, the difference between $\mathrm{Mgb}$ and $C K-M B$ sensitivities was 39\%, (CI 9\% to 69\%). The difference between $\mathrm{Mgb}$ and CK especificities was $13 \%$, (CI 12\% to 14\%) and the difference between $M g b$ and $C K$ MB specificities was $2 \%$, (CI -5\% to $9 \%$ ).

Conclusion - In the studied population, Mgb was more sensitive than $C K-M B$ for the diagnosis of $M I$ with similar specificity and $M g b$ was more specific than $C K$ for this diagnosis.

Key-words: myocardial infarction diagnosis, myoglobin, creatine kinase

Arq Bras Cardiol, volume 70 (n 2), 75-80, 1998

Departamento de Clínica Médica da Universidade Federal de Santa Catarina, Florianópolis e Instituto de Cardiologia, São José

Correspondência: Alexandre Biasi Cavalcanti - Av. Jorn. Rubens A. Ramos, 2212/ 802 - 88015-702 - Florianópolis, SC

Recebido para publicação em 9/5/97

Aceito em 20/11/97
O diagnóstico de infarto agudo do miocárdio (IAM), baseado somente em critérios clínicos e eletrocardiográficos, pode ser difícil no momento em que o paciente é admitido à sala de emergência. Apenas 41\% a 56\% dos pacientes com IAM que chegam à emergência apresentam 
supradesnivelamento do segmento ST, sendo possível nesses casos estabelecer o diagnóstico de imediato ${ }^{1-3}$. Nos demais pacientes ocorrem alterações não diagnósticas, como bloqueio de ramo esquerdo, inversão de onda $\mathrm{T}$, infradesnivelamento de segmento ST ou mesmo eletrocardiograma $(\mathrm{ECG})$ normal $^{1-6}$.

Um passo importante na confirmação ou exclusão de infarto nesse grupo de pacientes é a medida das enzimas miocárdicas no soro. Entretanto as enzimas mais frequientemente utilizadas no diagnóstico de IAM, creatinoquinase total (CK) e sua fração MB (CK-MB), não apresentam elevação sérica até a $4^{\mathrm{a}}$ hora após o início da dor torácica e, portanto, não apresentam boa sensibilidade no estágio inicial do $\mathrm{IAM}^{7}$.

A mioglobina é uma hemeproteína de 17700 dáltons, encontrada no músculo cardíaco e esquelético. Seu peso molecular bastante baixo permite que seja um dos primeiros marcadores séricos identificáveis após lesão isquêmica do miocárdio ${ }^{8-10}$. Apesar do interesse na mioglobina como marcador precoce de IAM existir há alguns anos, apenas recentemente foram desenvolvidos métodos de dosagem capazes de fornecer medidas quantitativas de mioglobina sérica de modo rápido, viabilizando seu uso no ambiente de emergência ${ }^{11}$.

O presente estudo objetiva comparar o desempenho, em termos de teste diagnóstico, da dosagem de mioglobina sérica com a de CK e CK-MB, para o diagnóstico de IAM, numa amostra de pacientes com dor torácica.

\section{Métodos}

Trata-se de estudo observacional, prospectivo, não controlado e individual. Foram analisados 64 pacientes, admitidos no período compreendido entre 19/9/94 e 21/2/95, na emergência do Instituto de Cardiologia, anexo ao Hospital Regional em São José-SC, apresentando-se com até $6 \mathrm{~h}$ de dor torácica, sugestiva de IAM, não originada por trauma. Foram excluídos do estudo pacientes com dor torácica há mais de $6 \mathrm{~h}$ ou por tempo indeterminado, pacientes com história de trauma muscular, ressuscitação cardiopulmonar e insuficiência renal.

As informações pertinentes ao estudo foram colhidas pelo cardiologista ou médico residente em cardiologia no momento do atendimento e registradas em ficha de coleta de dados. O diagnóstico final foi obtido, posteriormente, através das informações colhidas nos prontuários dos pacientes. O diagnóstico de IAM foi estabelecido por cardiologistas da UTI coronária, e ulteriormente validado pelos autores, quando dois dos seguintes critérios tenham sido preenchidos: 1) história de desconforto torácico prolongado (>20min), não aliviado por nitrato sublingual; 2) alterações na evolução eletrocardiográfica consistentes com necrose (desenvolvimento de ondas Q); 3) mensurações seriadas tardias de CK e CK-MB elevadas ${ }^{12}$.

Para análise do desempenho dos marcadores, CK, CKMB e mioglobina, foi colhida uma amostra de sangue na admissão. Outras amostras de sangue foram colhidas, con- forme a rotina do serviço, porém apenas com a finalidade de estabelecer o diagnóstico dos pacientes. Os clínicos envolvidos no atendimento aos pacientes não tiveram acesso às dosagens de mioglobina.

A dosagem de mioglobina sérica foi realizada através de ensaios para mioglobina $\mathrm{N}$ latex myoglobin reagents ${ }^{\circledR}$ (da Behringwerk AG, Marburg, Alemanha), para uso com o nefelômetro Behring ${ }^{\circledR}$. Esse ensaio é baseado em partículas de poliestireno cobertas com anticorpos anti-mioglobina humana de coelhos. Em reação imunoquímica essas partículas aglutinam com a mioglobina plasmática emitindo fluorescência. A intensidade da fluorescência emitida é mensurada pelo nefelômetro e é proporcional à concentração de mioglobina na amostra. Resultados quantitativos são fornecidos em menos de $15 \mathrm{~min}$. Esse teste mede valores de mioglobina que vão de $25 \mu \mathrm{g} / \mathrm{L} \mathrm{a} 400 \mu \mathrm{g} / \mathrm{L}^{13}$. Como limite superior de referência foi adotado o valor de $70 \mathrm{mg} / \mathrm{L}^{11}$.

$\mathrm{A}$ atividade de $\mathrm{CK}$ total e sua fração $\mathrm{MB}$ foi mensurada a $25^{\circ} \mathrm{C}$ com kits Biodiagnóstica ${ }^{\circledR}$ e Merck $^{\circledR}$, respectivamente. A atividade de CK-MB foi medida por meio de imunoinibição baseada na presença de anticorpos inibidores anti-creatinoquinase M. Valores superiores a $70 \mathrm{U} /$ $\mathrm{L}$ para pacientes do sexo masculino e $58 \mathrm{U} / \mathrm{L}$ para pacientes do sexo feminino foram considerados anormais para $\mathrm{CK}^{14}$. Valores de CK-MB maiores que 10U/Le com aumento maior que $6 \%$ na relação entre a fração $\mathrm{MB}$ e CK total foram considerados anormais para CK-MB ${ }^{7,15}$.

Com relação à análise dos dados, as variáveis categóricas (sexo e características da dor) foram expressas por suas freqüências (número e percentual). As variáveis contínuas (idade) foram expressas pelas médias e desvios padrão. Foi realizada comparação de características clínicas e demográficas entre grupos, com ausência ou presença de IAM. Para tanto foram empregados os testes de qui-quadrado para as variáveis categóricas, e t-Student para as contínuas, bem como calcularam-se as diferenças entre os valores das variáveis e os intervalos de confiança de 95\% (IC $95 \%$ ) para as diferenças ${ }^{16}$. Consideraram-se estatisticamente significativas as diferenças entre os grupos quando $\mathrm{P}<$ 0,05 ou o IC $95 \%$ não incluía o valor zero ${ }^{16,17}$.

Para avaliação do desempenho dos marcadores laboratoriais, CK, CK-MB e mioglobina, em relação ao diagnóstico de IAM, foram calculados sensibilidade, especificidade, valores preditivos positivo e negativo, acurácia

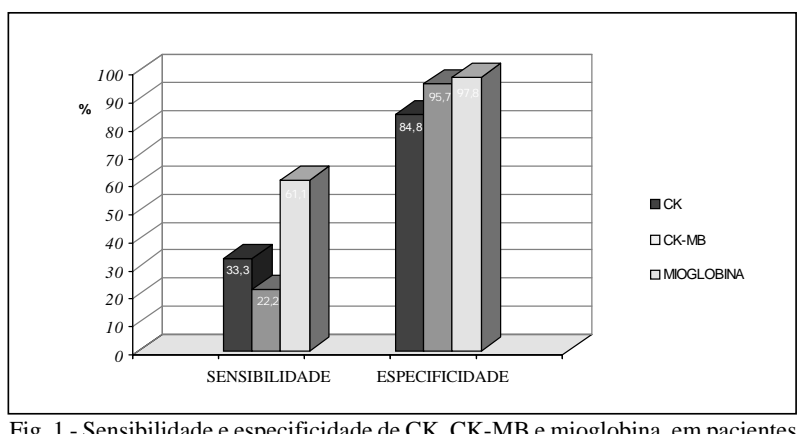

admitidos com menos de $6 \mathrm{~h}$ de dor torácica (a média do tempo de dor foi de $3,6 \mathrm{~h}$ ). 


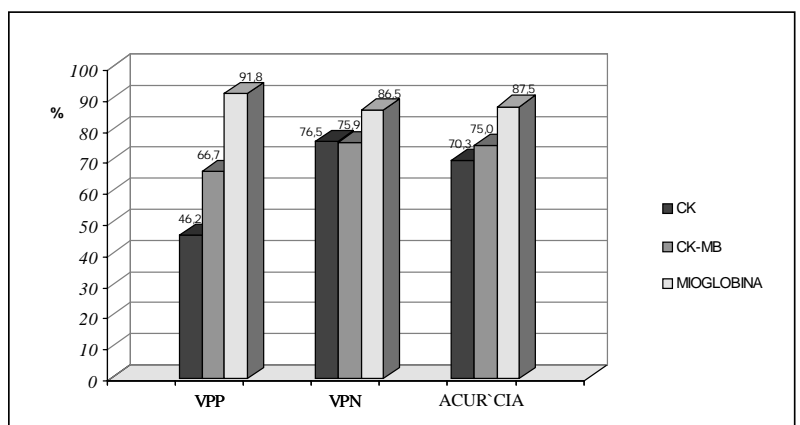

Fig. 2 - Valores preditivo positivo (VPP), negativo (VPN) e acurácia de CK, CK-MB e mioglobina, em pacientes admitidos com menos de seis horas de dor torácica (a média do tempo de dor foi de $3,6 \mathrm{~h})$.

e razão de verossimilhança positiva ${ }^{18}$. A razão de verossimilhança positiva, que é a tradução do termo inglês likelihood ratio, significa a chance, em número de vezes, que tem um paciente com exame alterado de estar acometido pela doença, em relação a um paciente com exame normal ${ }^{18}$. Foi definida a razão de verossimilhança pela fórmula: razão de verossimilhança positiva $=($ pacientes com exame positivo e com IAM / pacientes com IAM / (pacientes com exame positivo e sem IAM / pacientes sem IAM).

Foi calculada a diferença entre a sensibilidade de mioglobina e CK, e entre a de mioglobina e CK-MB, bem como a diferença entre a especificidade de mioglobina e CK, e entre a de mioglobina e CK-MB. Para as diferenças obtidas foram calculados intervalos de confiança de $95 \%$, e também foi aplicado o teste de qui-quadrado. As diferenças entre os grupos foram consideradas estatisticamente significativas quando $\mathrm{P}<0,05$ ou o IC $95 \%$ não incluía o valor zero $^{16,17}$.

\section{Resultados}

No período do estudo foram incluídos, inicialmente, 102 pacientes. Desses foram excluídos 38 pacientes. Seis por que o tempo transcorrido, desde o início dos sintomas até a admissão, não pôde ser determinado; quatro por que tiveram suas fichas de coleta de dados preenchidas incompletamente e 28 foram excluídos por apresentarem tempo de sintomas superior a $6 \mathrm{~h}$. Nenhum paciente apresentava história de trauma muscular, ressuscitação cardiopulmonar ou insuficiência renal. Portanto restaram 64 pacientes que observavam os critérios de inclusão no estudo.

A média de tempo transcorrido entre o início dos sintomas e a coleta das amostras de sangue para dosagem dos marcadores, foi de 3,6h (1-6h). Após a coleta das amostras de sangue, a dosagem dos marcadores processou-se dentro de $15 \mathrm{~min}$, em média.

Dos 64 pacientes, foi estabelecido diagnóstico de IAM em $18(28,1 \%)$. Nos pacientes restantes $(71,9 \%)$, o diagnóstico foi de angina de peito ou dor de origem extracardíaca. Entre os pacientes com IAM, $13(72,2 \%)$ foram com onda $\mathrm{Q}$, e cinco sem onda $\mathrm{Q}(27,8 \%)$.

Quanto às características clínicas dos grupos de pacientes, com e sem IAM, nota-se que a presença de pacientes

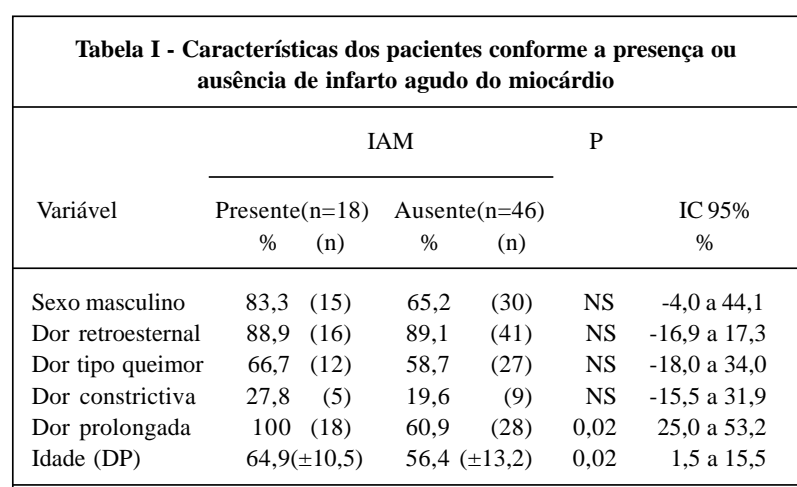

Fonte: ficha de coleta de dados. Instituto de Cardiologia da Secretaria de Estado da Saúde, 1995; IAM- infarto agudo do miocárdio. Entre parênteses estão os números absolutos de pacientes, exceto em idade em que o desvio padrão aparece entre parênteses. NS- não significativo (P>0,05); IC 95\%- intervalo de confiança de $95 \%$ para as diferenças entre o valor da variável com IAM presente ou ausente.

do sexo masculino, com dor tipo queimor ou constrictiva foi mais prevalente no grupo com IAM, porém não houve diferença estatisticamente significativa. A presença de dor retroesternal foi mais prevalente no grupo sem IAM, contudo, sem significado estatístico. A média de idade e a prevalência de dor prolongada (duração >20min), nos pacientes com IAM, foi superior à dos pacientes sem IAM, sendo a diferença estatisticamente significativa (tab. I).

Dentre os pacientes com IAM $(\mathrm{n}=18)$, houve seis com valor de CK alterado (até $6 \mathrm{~h}$ de sintomas). No grupo de pacientes sem IAM ( $n=46)$, houve 39 resultados normais (tab. II). A sensibilidade calculada para CK foi de $33,3 \%$, e a especificidade $84,8 \%$ (fig. 1 ). O valor preditivo positivo foi de $46,2 \%$, o valor preditivo negativo de $76,5 \%$, a acurácia de 70,3\% e a razão de verossimilhança positiva de 2,2 (fig. 2).

A CK-MB apresentou valores alterados em quatro dos pacientes com IAM $(n=18)$ e valores normais em 44 dos pacientes sem IAM ( $\mathrm{n}=46$ ), (até $6 \mathrm{~h}$ de sintomas), (tab. II). A sensibilidade de CK-MB foi de $22,2 \%$, e a especificidade de $95,7 \%$ (fig. 1). O valor preditivo positivo foi de $66,7 \%$, o valor preditivo negativo $75,9 \%$, a acurácia $75,0 \%$ e a razão de verossimilhança positiva 5,1 (fig. 2).

\begin{tabular}{|c|c|c|c|c|c|c|}
\hline \multicolumn{7}{|c|}{$\begin{array}{c}\text { Tabela II - Distribuição dos pacientes com dor torácica, não traumática, } \\
\text { com menos de } 6 \text { h de duração, conforme a presença ou ausência de } \\
\text { infarto agudo do miocárdio e resultado de creatinofosfoquinase } \\
\text { normal ou alterado }\end{array}$} \\
\hline \multirow[b]{2}{*}{$\begin{array}{l}\text { Resultado do } \\
\text { Teste }\end{array}$} & \multicolumn{3}{|c|}{ IAM Presente } & \multicolumn{3}{|c|}{ IAM Ausente } \\
\hline & CK & CK-MB & Mgb & CK & CK-MB & $\mathrm{Mgb}$ \\
\hline Alterado & 6 & 4 & 11 & 7 & 2 & 1 \\
\hline Normal & 12 & 14 & 7 & 39 & 44 & 45 \\
\hline Total & & 18 & & & 46 & \\
\hline \multicolumn{7}{|c|}{$\begin{array}{l}\text { Fonte: ficha de coleta de dados. Instituto de Cardiologia da Secretaria de Es- } \\
\text { tado da Saúde, } 1995 \text {. IAM- infarto agudo do miocárdio; CK- creatino- } \\
\text { fosfoquinase; CK-MB- fração MB da CK; Mgb- mioglobina. O tempo médio } \\
\text { transcorrido entre o início dos sintomas e a coleta dos marcadores foi de 3,6h. } \\
\text { Os valores de corte para CK foram 58U/L para mulheres e 70U/L para homens; } \\
\text { para CK-MB foi 10U/L com relação CK-MB/CK total >6; para mioglobina foi } \\
70 \mu \mathrm{g} / \mathrm{L} \text {. }\end{array}$} \\
\hline
\end{tabular}




\begin{tabular}{|c|c|c|c|}
\hline \multicolumn{4}{|c|}{$\begin{array}{l}\text { Tabela III - Diferenças obtidas entre mioglobina e creatinofosfoquinase, } \\
\text { e entre mioglobina e creatinofosfo-quinase fração MB quanto aos testes } \\
\text { de sensibilidade e especificidade e os respectivos intervalos de confiança } \\
\text { de } 95 \% \text { e valores P }\end{array}$} \\
\hline \multirow[t]{2}{*}{ Teste } & & & n \\
\hline & \multicolumn{3}{|c|}{ Mioglobina - CK-MB } \\
\hline \multirow[t]{2}{*}{ Sensibilidade } & & & \\
\hline & & $-3,5$ a 5 & a $68,5(\mathrm{P}<$ \\
\hline \multirow[t]{2}{*}{ Especificidade } & & 13 & 2,1 \\
\hline & & 12,4 a $13,6(\mathrm{P}<0,05)$ & $-5,1$ a 9,3 (NS) \\
\hline \multicolumn{4}{|c|}{$\begin{array}{l}\text { Fonte: ficha de coleta de dados. Instituto de Cardiologia da Secretaria de Esta- } \\
\text { do da Saúde, } 1995 \text {. CK- creatinofosfoquinase e CK-MB sua fração MB; IC } 95 \% \text { - } \\
\text { intervalo de confiança de } 95 \% \text {, para proporções, obtido para as diferenças. Todos } \\
\text { os valores são percentagens, exceto valor P. }\end{array}$} \\
\hline
\end{tabular}

No grupo de pacientes com IAM ( $\mathrm{n}=18), 11$ apresentaram valores alterados de mioglobina (até $6 \mathrm{~h}$ de sintomas). No grupo de pacientes sem IAM ( $n=46), 45$ apresentaram valores normais de mioglobina (tab. II). A mioglobina apresentou sensibilidade de $61,1 \%$ e especificidade de $97,8 \%$ (fig. 1). O valor preditivo positivo foi de $91,8 \%$, o valor preditivo negativo $86,5 \%$, a acurácia $87,5 \%$ e a razão de verossimilança positiva 28,1 (fig. 2).

A diferença entre a sensibilidade de mioglobina e a de CK foi de 27,8\%, e o intervalo de confiança de $95 \%$ (IC 95\%), obtido para esta diferença, de $-3,5 \%$ a $59,1 \%$ (P=NS). A diferença entre a sensibilidade de mioglobina e a de CKMB foi de 38,9\%, e o IC 95\% de 9,3\% a 68,5\% (P<0,05). A diferença entre a especificidade de mioglobina e a de CK é de $13,0 \%$, e o IC $95 \%$ foi de $12,4 \%$ a $13,6 \%$ (P $<0,05)$. Subtraindose a especificidade de mioglobina da de CK-MB obteve-se $2,1 \%$, e o IC de $95 \%$ de $-5,1 \%$ a $9,3 \%$ (P=NS) (tab. III).

\section{Discussão}

A partir de critérios clínicos e eletrocardiográficos, aproximadamente, $40 \%$ a 55\% dos pacientes que chegam à emergência, com dor torácica, não traumática, ficam com diagnóstico indefinido ${ }^{1-3}$. As enzimas tradicionalmente usadas no diagnóstico de IAM, CK e CK-MB, demoram para se elevar ${ }^{7}$. Dessa maneira, existe carência de meios para estabelecer diagnóstico nesse grupo grande de pacientes.

A necessidade de se estabelecer diagnóstico de IAM logo que o paciente é admitido à emergência tem suscitado interesse sobre marcadores biológicos detectáveis nas primeiras horas da doença. Vários estudos mostram que a mioglobina é um marcador sérico identificável nas primeiras horas de IAM ${ }^{8-10}$, apresentando alta sensibilidade em sua detecção precoce. Enquanto que as enzimas CK e CK-MB demoram usualmente 4 a $8 \mathrm{~h}$ para se elevar ${ }^{7}$, a mioglobina eleva-se em média em $2,5 \mathrm{~h}$ e retorna a valores normais entre $24 \mathrm{e} 48 \mathrm{~h}^{9}$.

Neste estudo, a mioglobina foi comparada com CK e CK-MB, tendo obtido o melhor desempenho, apresentando valores superiores ou semelhantes aos de CK e CK-MB em todos os testes realizados. A sensibilidade obtida para a mioglobina foi mais alta que a de CK-MB, e a diferença foi estatisticamente significativa, evidenciando uma melhor capacidade de deteç̧ão do diagnóstico de IAM no paciente que é admitido à emergência com dor torácica. Quanto à especificidade, a de mioglobina foi superior à de CK-MB, porém sem diferença estatisticamente significativa (fig. $1 \mathrm{e}$ 2, tab. III).

Em relação a CK, a mioglobina mostrou valor maior de sensibilidade, porém com diferença sem significado estatístico. Porém a especificidade da mioglobina foi significativamente superior à de CK. Este fenômeno provavelmente ocorreu devido aos valores de corte baixos que foram usados para $\mathrm{CK}$, que ganhou sensibilidade às custas de redução em sua especificidade (fig. 1, tab. III).

Os valores preditivo positivo e negativo, e a acurácia também foram maiores para a mioglobina. A razão de verossimilhança positiva foi várias vezes superior para mioglobina do que para os outros marcadores (fig. 2).

O quadro 1 mostra os valores de sensibilidade e especificidade obtidos por outros autores em estudos que comparam CK, CK-MB e mioglobina, no diagnóstico de IAM $^{8,11,19-25}$. Observa-se grande variabilidade nos valores obtidos, entre os diferentes estudos, que é mais notória em relação aos valores de sensibilidade. Alguns fatores podem explicar esse fenômeno, porém a principal causa da grande variação obtida nos valores de sensibilidade é o fator tempo. A elevação dos marcadores de infarto no plasma é tempodependente, ocorrendo sua elevação acima de valores normais vários minutos após a oclusão arterial, elevação progressiva até o pico plasmático, e após decréscimo até a normalização ${ }^{7}$. Dessa maneira, a sensibilidade dos marcadores, também, irá variar em função do tempo, apresentando, nas primeiras horas de IAM, elevação progressiva, conforme aumenta a média do tempo de sintomas de um dado grupo de pacientes ${ }^{26}$. Além do fator tempo, que foi diferente entre os vários estudos mostrados, variações nos métodos de dosagem, valores de corte e critérios para diagnóstico de IAM, também podem ter sido responsáveis pela variação nos valores de sensibilidade e especificidade encontrados, quando se comparam os diferentes estudos.

A despeito da diversidade encontrada nos testes de sensibilidade e especificidade para os marcadores, entre os diferentes estudos, observa-se que em todos eles a sensibilidade da mioglobina foi superior à da CK e da CK-MB. Quanto à especificidade, valores semelhantes foram encontrados para os três marcadores, com exceção feita ao estudo de Montague e Kircher ${ }^{24}$. Tais resultados, especialmente quando se atenta para estudos com maior número de pacientes ${ }^{19,22,23}$, corroboram os resultados do presente estudo e destacam o grande valor que pode ter a mioglobina como exame para o diagnóstico de IAM.

A aplicação de um exame sensível e com especificidade próxima de $100 \%$ na detecção de IAM tem grande valor, especialmente quando não ocorre supradesnivelamento do segmento ST no ECG de admissão do paciente, pois o reconhecimento desta enfermidade é fundamental para a tomada de decisões acerca do manuseio do paciente a curto e a longo prazo. Estudos mostram que 1,9\% a $8 \%$ dos 


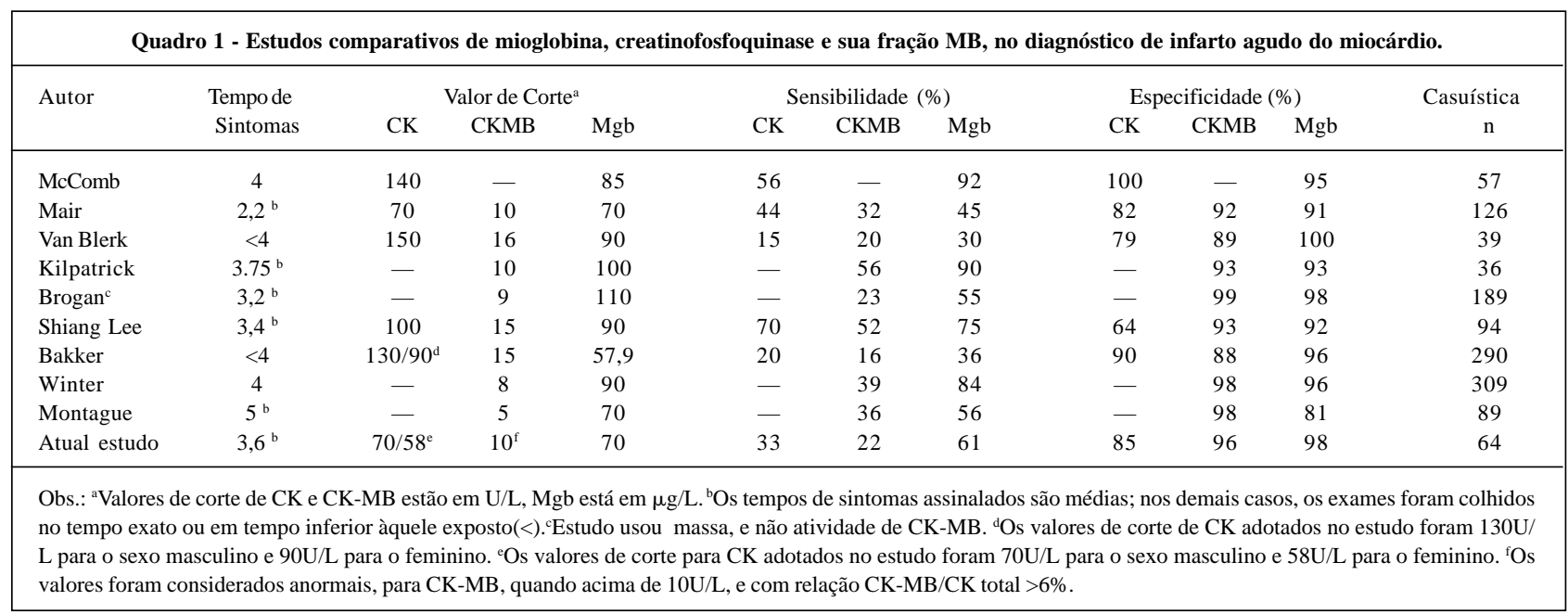

pacientes com IAM são, inapropriadamente, diagnosticados como tendo outras doenças, geralmente não relacionadas à isquemia coronária e, por isso, são dispensados da emergência ${ }^{19}$. Mesmo que esses pacientes liberados tenham sintomas menos intensos, sua mortalidade é maior do que naqueles admitidos em unidades coronárias ${ }^{27}$. Também entre pacientes admitidos com insuficiência coronária é importante saber quais têm IAM, pois esses pacientes apresentam maior mortalidade a curto prazo ${ }^{28}$.

$\mathrm{O}$ advento de novas técnicas de restabelecimento do fluxo coronário após o infarto, principalmente trombólise e angioplastia, reitera a urgência na realização de diagnóstico em pacientes com dor torácica, pois, sabe-se, que quanto mais precoce foi a recanalização, tanto mais benefícios receberão ${ }^{29-31}$. No entanto apenas pequena fração de pacientes infartados é submetida a trombólise ou angioplastia atualmente ${ }^{5,32,33}$. Um exame que detecte IAM precocemente poderia ajudar no momento de se indicar trombólise, aumentando o número de pacientes beneficiados pela mesma ${ }^{34}$.

Com relação ao aspecto custo dos kits, os exames de CKe CK-MB custam $R \$ 0,25$ e $R \$ 1,20$ por exame, respectivamente. Atualmente são mais acessíveis do que os kits para dosagens de mioglobina, que custam $R \$ 24,00$ por exame. A aparelhagem necessária para a realização dos exames (nefelômetro) é a mesma empregada na dosagem de várias outras proteínas plasmáticas, por isso já existente em vários laboratórios de análise.

A dosagem de mioglobina sérica, como exame no diagnóstico de IAM, não tem sido parte da prática médica atual. Contudo, na amostra de 64 pacientes estudados, que se apresentaram com dor torácica, durando menos que $6 \mathrm{~h}$, a mioglobina demonstrou ser mais sensível do que a CK-MB e com especificidade semelhante. Em relação a CK foi obtida sensibilidade semelhante e especificidade superior para mioglobina. Os valores preditivo positivo e negativo, acurácia e razão de verossimilhança positiva foram maiores para mioglobina do que para CKe CK-MB.

A falta de conhecimento a respeito da mioglobina e a existência de tabus como a sua suposta baixa especificidade têm contribuído para o pouco emprego deste marcador. $\mathrm{O}$ conhecimento das características da mioglobina como marcador mais precoce de IAM introduz valioso exame laboratorial na prática médica, como mais um instrumento à disposição do clínico, para a tomada da decisão diagnóstica.

\section{Agradecimentos}

AoSrs. AdolfoHaruo Koga, Danielle Legat Albino, Carlos AlbertoLira Pamplonae demais funcionários do laboratório do Hospital Regional de São José, pela obtenção dos dados laboratoriais e ao Dr. Miguel de Patta, pela colaboração prestada.

\section{Referências}

1. Lee TH, Rouan GW, Weisberg MC et al. - Sensitivity of routine clinical criteria for diagnosing myocardial infarction within 24 hours of hospitalization. Ann Intern Med 1987; $106:$ 181-6.

2. Cragg DR, Friedman HZ, Bonema JDet al-Outcome of patients with acute myocardial infarction who are ineligible for thrombolytic therapy. Ann Intern Med $1991 ; 115: 173-7$.

3. ISIS-2 (Second International Study of Infarct Survival) Collaborative Group Randomised trial of intravenous streptokinase, oral aspirin, both, or neither among 17187 cases of suspected acute myocardial infarction: ISIS-2. Lancet $1988 ; 2: 349-60$

4. Rude RE, Poole WK, Muller JE et al. - Electrocardiographic and clinical criteria for recognition of myocardial infarction based on analysis of 3,697 patients. Am J Cardiol 1983; 52: 936-42.

5. Karlson BW, Herlitz J, Edvardsson N, Emanuelsson H, Sjölin M, Hjalmarson A - Eligibility for intravenous thrombolysis in suspected acute myocardial infarction. Circulation 1990; 82: 1140-6.

6. Karlson BW, Herlitz J, Wicklund O, Richter A, Hjalmarson A - Early prediction of acute myocardial infarction from clinical history, examination and electrocardiogram in the emergency room. Am J Cardiol 1991; 68: 171-5.

7. Lee TH, Goldman L-Serum enzyme assays in the diagnosis of acute myocardial infarction. Ann Intern Med 1986; 105: 221-33

8. McComb JM, McMasterEA, MacKenzie G, Adgey AAJ - Myoglobin and creat- 
ine kinase in acute myocardial infarction. Br Heart J 1984; 51: 189-94.

9. Drexel H, Dworzack E, Kirchmair W, Milz MM, Puschendorf B, Dienstl F Myoglobinemia in the early phase of acute myocardial infarction. Am Heart J 1983; 105: 642-51

10. Gilkeson G, Stone MJ, Waterman M et al. - Detection of myoglobin by radio immunossay in human sera: its usefulness and limitations as an emergency room screening test for acute myocardial infarction. Am Heart J 1978; 95: 70-7.

11. Mair J, Artner-Dworzak E, Lechleitner P et al. - Early diagnosis of acute myocardial infarction by a newly developed rapid immunoturbidimetric assay for myoglobin. Br Heart J 1992; 68 : 462-8

12. Reiss C, Eisenberg P - Cardiopatia isquêmica. In: Woodley M, Whelan A Manual de Terapêutica Clínica. 27 ed. Rio de Janeiro: Medsi, 1994: 112.

13. Massoubre C, Chivot L, Mainard F, Bridji B, Madec Y - Immunonephelometric assay of myoglobin. Clin Chim Acta 1991; 201: 223-30.

14. Szasz G-Laboratory measurement of creatine kinase activity. In: Szasz G - Proceedings: Second International Symposium on Clinical Enzymology. $3^{\text {rd }}$ ed. Chicago: 1975.

15. Würzburg U, Hennrich N, Orth HD et al - Quantitative determination of creatine kinase isoenzyme catalytic concentrations in serum using immunological methods. J Clin Chem Clin Biochem 1977; 15: 131-7.

16. Gardner MJ, Altman D - Confidence intervals rather than P values: estimation rather than hypothesis testing. Br Med J 1986; 282: 746-50.

17. Chance WA - Tests of hypothesis, part one. In: Chance WA-Statistical Methods for Decision Making. 1 ${ }^{\text {st }}$ ed. New Haven: Richard D. Irwin, 1969: 41.

18. Jaeschke R, Guyatt GH, Sackett DL- Users' guides to the medical literature: III How to use an article about a diagnostic test: B. What are the results and will they help me in caring for my patients? JAMA 1994; 271: 703-7.

19. Brogan GX Jr, Friedman S, McCuskey Cet al - Evaluation of a new rapid quantitative immunoassay for serum myoglobin versus CK-MB for ruling out acute myocardial infarction in the emergency department. Ann Emerg Med 1994; 24: 665-71.

20. Kilpatrick WS, Wosornu D, McGuiness JB, Glen ACA-Early diagnosis of acute myocardial infarction: CK-MB and myoglobin compared. Ann Clin Biochem 1993; 30: 435-8.

21. LeeHS, Cross SJ, GarthwaithPetal-Comparison of the value of novel rapidmeasurement of myoglobin, creatine kinase, and creatine kinase MB with the electrocardiogram for the diagnosis of acute myocardial infarction. Br Heart J 1994; 71: 311-5.
22. Bakker AJ, Koelemay MJW, Gorgels JPM et al - Troponin T and myoglobin at admission: value of early diagnosis of acute myocardial infarction. Eur Heart $\mathrm{J}$ 1994; 15: 45-53.

23. Winter RJ, Koster RW, Sturk A, Sanders GT - Value of myoglobin, troponin T, and CK-MB mass in rulling out an acute myocardial infarction in the emergency room. Circulation 1995; 92: 3401-7.

24. Montague C, Kircher T - Myoglobin in the early evaluation of acute chest pain. Am J Clin Pathol 1995; 104: 472-6.

25. Van Blerk M, Maes V, Huyghens L, Derde MP, Meert R, Gorus FK - Analytical and clinical evaluation of creatine kinase MB mass assay by Imx: comparison with MB isoenzyme activity and serum myoglobin for early diagnosis of myocardial infarction. Clin Chem 1992; 38: 2380-6.

26. Sox HC - Probability theory in the use of diagnostic tests. Ann Intern Med 1983; 104: 60-6.

27. Lee TH, Brand D, Weisberg M, Acampora D, Rouan G, Goldman L-Patients sent home from emergency rooms with myocardial infarction : clinical characteristics and implications. Clinical Research 1985; 33: 257A.

28. Schroeder JS, Lamb IH, Hu M - Do patients in whom myocardial infarction has been ruled out have a better prognosis after hospitalization than those surviving infarction? N Engl J Med 1980; 303: 1-5.

29. Tate DA, Dehmer GJ - New challenges for thrombolytic therapy. Ann Intern Med 1989; 110: 953-6.

30. Rozenman Y, Gotsman MS - The earliest diagnosis of acute myocardial infarction. Annu Rev Med 1994; 45: 31-44.

31. Roberts R, Kleiman NS - Earlier diagnosis and treatment of acute myocardial infarction necessitates the need for a new diagnostic mind-set. Circulation 1994; 89: 872-81.

32. Thomas HL, Weisberg MC, Brand DA, Rouan GW, Goldman L - Candidates for thrombolysis among emergency room patients with acute chest pain. Ann Intern Med 1989; $110: 957-62$

33. Althouse R, Maynard C, Cerqueira MD, Olsufka M, Ritchie JL, Kennedy JD - The western Washington myocardial infarction registry and emergency department tissue plasminogen activator treatment trial. Am J Cardiol 1990; 66: 1289-303.

34. Haastrup B, Gill S, Haghfelt T - Thrombolysis in acute myocardial infarction: the implementation of thrombolytic therapy in a coronary care unit in 1992. Cardiology 1994; 85: 397-406. 